\title{
Altered Motivation and Learning Following Opiate Withdrawal: Evidence for Prolonged Dysregulation of Reward Processing
}

\author{
Glenda C Harris' and Gary Aston-Jones*,', \\ 'Department of Psychiatry, University of Pennsylvania, Philadelphia, PA, USA
}

\begin{abstract}
Opiate abuse has been associated with cognitive deficits in human addicts. To determine if prior opiate exposure alters the ability to learn, we trained animals in an instrumental learning task for a food reward. During a 2-week period after withdrawal, morphine-abstinent rats were significantly slower at learning an escalating fixed-ratio response for food reward compared to placebo-treated animals. When these same animals were trained in a conditioned suppression paradigm (two tone-shock pairings given in the operant box), the morphinewithdrawn animals showed greater retention by taking significantly longer to resume responding for food reward when the tone was presented. In a third experiment, morphine-abstinent rats withdrawn 2 or 5 weeks were tested for their ability to associate a highly palatable food reward with a specific environment using a place-conditioning paradigm. At 2 weeks postwithdrawal, morphine-abstinent rats did not show any significant place preference for a food they readily consumed, while placebo-treated rats readily learned to prefer the food-paired environment. At 5 weeks postwithdrawal, rats developed significantly less preference for food-associated cues, but more preference for morphine-associated cues, compared to placebo-treated animals. These data suggest that prior morphine exposure may have prolonged effects on the motivation for natural rewards, which in turn may compromise the ability of former addicts to overcome their addictions. Neuropsychopharmacology (2003) 28, 865-87I, advance online publication, 12 March 2003; doi:I 0. I038/sj.npp. I 300122
\end{abstract}

Keywords: morphine; morphine dependence; conditioned place preference; food conditioning; instrumental conditioning; conditioned suppression

\section{INTRODUCTION}

A recent study reported cognitive deficits in human heroin addicts similar to those seen in patients with selective lesions of the temporal and frontal lobes (Ornstein et al, 2000). Brain imaging studies involving human opiate addicts have indicated that many brain areas, particularly the frontal and temporal lobes, are hypofunctional during both prolonged abstinence (Gerra et al, 1998) and acute withdrawal (Krystal et al, 1995). The suppressed activity in these cortical and limbic areas may relate to the cognitive deficits found, as well as to the symptoms of anxiety and depression that linger after the physical symptoms of withdrawal have dissipated (Jaffe, 1990).

It has been reported that generalized dysphoria and depressive symptoms can be present for weeks or months following abstinence from opiates (Dole et al, 1966; Martin and Jasinski, 1969). Many researchers have hypothesized that these symptoms of protracted opiate withdrawal involve a dysregulation of hedonic processing because of

\footnotetext{
*Correspondence: Dr G Aston-Jones, Department of Psychiatry, University of Pennsylvania, 705 Stellar Chance/6100 Philadelphia, PA 19104, USA, Tel: +1 215573 5200, Fax: + 215573 5202, E-mail: gaj@mail.med.upenn.edu

Received 02 August 2002; revised 22 November 2002; accepted 27 November 2002
}

alterations in brain chemistry (Solomon, 1977; Koob and Le Moal, 1997). If opiate-withdrawn animals are dysfunctional in processing hedonic events, then it could be predicted that the withdrawn animals would have problems learning about appetitive reinforcers that predict pleasurable events, but not about other types of reinforcement (ie aversive events).

In this series of experiments, we used several paradigms to determine whether opiate-abstinent animals show altered learning. In Experiment 1, rats were trained to lever press for food on an escalating fixed-ratio schedule of reward during a 2-week period postmorphine withdrawal. In Experiment 2, these same animals were used in a conditioned suppression paradigm, in which a tone previously paired with foot shock was presented during the performance of the instrumental food task to assess the effect on responding (Experiment 2). In Experiment 3, we examined food place preference at 2 and 5 weeks postmorphine withdrawal and compared this to morphine place preference.

\section{MATERIALS AND METHODS}

\section{Subjects}

Male Sprague-Dawley rats (200-250 g) from Harlan (Indianapolis, IN) were used in all experiments. Rats were 
group housed in accordance with NIH guidelines on a 12-h light/dark cycle with food and water available ad libitum. All animal procedures were approved by the Institutional Animal Care and Use Committees of the Philadelphia Veterans Administration Medical Center and the University of Pennsylvania. A total of 45 animals were used with individual group numbers of seven to eight animals.

\section{Chronic Drug Treatment}

Two $75 \mathrm{mg}$ morphine tablets (provided by the National Institute on Drug Abuse) were subcutaneously implanted under halothane anesthesia to induce morphine dependence. Nondependent rats were implanted at the same time with inert placebo pellets. All chronically morphinepretreated animals are denoted by the abbreviation ' $M$ ', and all placebo-pretreated animals are denoted by the abbreviation ' $\mathrm{P}$ '. Previous studies have shown that morphine pellets are a reliable way to induce physical dependence (Yoburn et al, 1985; Gold et al, 1994). The signs of physical dependence begin to wane around day 14 as the morphine pellets dissolve (Gold et al, 1994). Deprivation withdrawal (a model of abstinence) was induced by removing the pellets after 14 days.

\section{Operant Food-Conditioning Procedure}

Rats were subchronically pretreated with either morphine or placebo pellets, as described above ( $n=7$ each). At 1 week after pellet removal, rats were placed on a food deprivation schedule, such that they received only $15 \mathrm{~g}$ of food daily, sufficient to maintain them at $\sim 85 \%$ of their free-feeding weight. The animals were fed each day after the training sessions. Training and testing took place in Med Associates operant chambers housed within sound- and light-resistant shells. Each chamber was equipped with a pellet dispenser that delivered one $45 \mathrm{mg}$ Noyes pellet (formula A/I) when activated. The chamber contained one active lever on which responses were reinforced with one pellet of food and the illumination of a light above the active lever, and one inactive lever on which responses were never reinforced. A $3 \mathrm{~W}$ house light mounted on the top-center of the wall opposite the magazine provided illumination. Microcomputers equipped with the MED-PC program (Med Associates) controlled the equipment and recorded lever presses.

At $24 \mathrm{~h}$ after starting the food deprivation schedule, rats were placed in the operant chamber and allowed to bar press for food on a fixed-ratio schedule of one response per one reinforcer (FR1). During the first day of training, pellets were taped to the lever, and rats received a free pellet every $5 \mathrm{~min}$ to facilitate learning. Subsequent training sessions were $30 \mathrm{~min}$ long and rats were given two sessions per day until they reached a criterion of 40 reinforcers in a $30 \mathrm{~min}$ time period. Once this criterion was maintained for two consecutive sessions, rats were placed on progressively more difficult schedules of reinforcement (FR2, FR4, FR8) until they mastered an FR16 schedule. The amount of time (in minutes) it took each rat to reach the criterion of two consecutive sessions with 40 reinforcers was recorded.

\section{Conditioned Suppression Paradigm}

Once all the animals mastered the FR16 schedule, they were conditioned by receiving two tone-shock pairings in the operant chamber. The CS was a $30-\mathrm{s} 1 \mathrm{kHz}$ tone and the US was a $1 \mathrm{~mA} / 0.5$-s foot shock. The day after this conditioning, the animals were placed back in the operant chamber, the CS was presented alone, and the amount of time it took for the animals to resume responding on the FR16 ratio was recorded for $30 \mathrm{~min}$. After 5 days, a second identical test took place.

\section{Conditioned Place Preference Procedure}

Rats were implanted with morphine $(n=16)$ or placebo pellets $(n=15)$, as described above. Pellets were removed after 14 days in all animals to initiate abstinence withdrawal. The place preference procedure was carried out in a Plexiglas apparatus consisting of two distinct compartments separated by a tunnel. One compartment had a grid floor with black walls, and the second compartment had a mesh floor with black and white stripes on the walls. Each compartment was equipped with photocells to record automatically the time that the animals spent in each compartment (MED Associates, East Fairfield, VT). On the first day after pellet removal, animals were allowed to explore freely all of the apparatus for $15 \mathrm{~min}$, and the amount of time spent in each compartment was recorded. None of the animals had an initial bias for either compartment, and were randomly assigned to one compartment for food conditioning in a balanced design. Animals received conditioning either 1 week or 4 weeks after pellet removal. By this time all somatic signs of opiate withdrawal had dissipated. Conditioning consisted of pairing one side of the chamber with 6-oat flavored pieces of Lucky Charms cereal (General Mills, Minneapolis, $\mathrm{MN})$. The other chamber had no food in it but contained an empty plastic dish similar to the one used to hold the cereal. Conditioning sessions occurred in the morning and afternoon and the presentation of the food was alternated between the morning and afternoon sessions on three separate days. Conditioning sessions were $30 \mathrm{~min}$ long and all rats consumed all the food presented during the sessions. Since the food was highly desirable, the rats were not food deprived for this experiment. Testing occurred 5 days following conditioning during which $M$ and $\mathrm{P}$ animals were given free access to the apparatus for $15 \mathrm{~min}$. The amount of time spent in each compartment was recorded.

\section{Morphine Conditioned Place Preference Procedure}

At 1 week after being placed back on a free feeding schedule, six $\mathrm{M}$ and six $\mathrm{P}$ rats from the operant foodtraining task were trained in a morphine place-conditioning task. Conditioning occurred at 4 weeks postmorphine withdrawal. The apparatus and procedure for this place conditioning was the same as described above for food conditioning. Conditioning took place over a 3-day period with alternating injections of morphine $(10 \mathrm{mg} / \mathrm{kg}$, i.p.) or saline given in $30 \mathrm{~min}$ morning and afternoon sessions. Testing took place 5 days after conditioning. 


\section{Data Analyses}

For both the operant food training data and conditioned suppression data, group differences in time were compared using a $t$-test. Place conditioning data were analyzed by calculating the time spent in the food-paired chamber minus the time spent in the other chamber. The resulting difference score was compared between groups using oneway analysis of variance. In addition, a within-group measurement of conditioned place preference was assessed by comparing the difference in time spent in the foodpaired and nonfood-paired sides preconditioning $v s$ postconditioning. Where necessary, post hoc analysis was carried out with a Newman-Keuls test.

\section{RESULTS}

\section{Experiment 1: Operant Food Training}

As shown in Figure 1 (upper panel), $\mathrm{M}$ animals took significantly more time to learn to bar press for food $(t(12)=2.4, p<0.015)$ and were significantly slower in mastering each FR requirement except for the transition between FR2 and FR4 (FR2: $t=2.65, p<0.01$; FR4: $t=1.20$, $p<0.12$; FR8: $t=2.79, p<0.008$; FR16: $t=2.28, p<0.02$ ) compared to $\mathrm{P}$ animals. To determine if the $\mathrm{M}$ rats were slower to learn because of a motor impairment, we compared response rates during the last $10 \mathrm{~min}$ of the session in which they reached the criterion to advance to a new FR schedule. Response rates for each group on the rewarded and nonrewarded levers are shown for each day of the experiment in Figure 1 (lower panel). As illustrated there, response rates are shifted to the right for the $M$ animals because they were slower to advance to the higher FR schedules. The $\mathrm{P}$ animals reached the criterion for the FR16 by day 10 , while the $M$ animals did not reach this level until day 12. However, there were no significant differences in response rates between $\mathrm{M}$ and $\mathrm{P}$ animals once they mastered an FR schedule (ie during the last 10 min of the session in which they reached the criterion to advance to a new FR schedule). This finding indicates that motor impairment is not a likely reason for the slower learning rates found in the $\mathrm{M}$ animals.

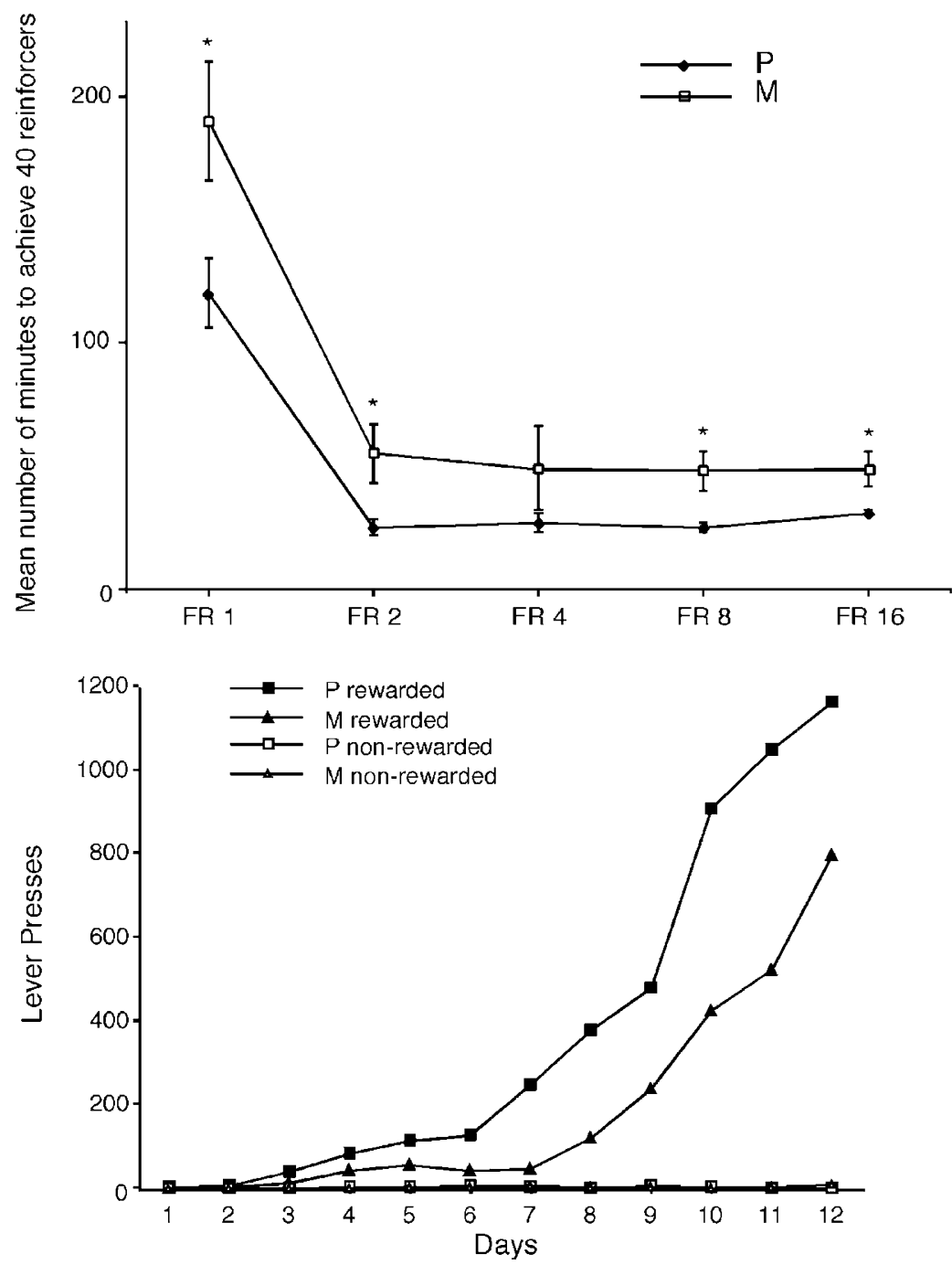

Figure I Acquisition of responding for food reward at each FR schedule. Upper panel: The mean amount of time for each group to achieve 40 reinforcers averaged over different 30-min sessions is depicted: *Significantly different $(p<0.05)$. Lower panel: Mean number of lever presses made by each group over the different days of training. $P$ animals complete the criterion for the FRI 6 by day 10. 


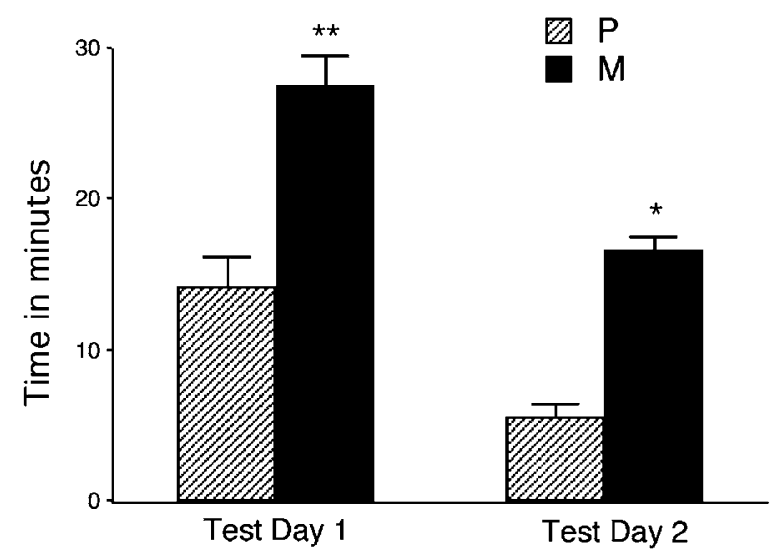

Figure 2 Mean amount of time to resume responding for food after the presentation of a tone that had been paired with shock for $M$ and $P$ groups. The maximum amount of time was $30 \mathrm{~min}$ : *Significantly different $(p<0.05)$, ${ }^{*}$ significantly different $(p<0.01)$.

\section{Experiment 2: Conditioned Suppression}

As can be seen from Figure 2, M animals took significantly longer to resume responding on an FR16 schedule for food after the presentation of the CS previously paired with shock on both test day $1(t(12)=4.1 p<0.01)$ and test day 2 $(t(12)=2.3 p<0.05)$. On test day 1 only two out of seven $\mathrm{M}$ animals resumed responding in the $30 \mathrm{~min}$ time period, while all seven of the $\mathrm{P}$ animals resumed responding. On test day 2, four of seven $\mathrm{M}$ animals resumed responding in the 30 min time period, compared to all seven $\mathrm{P}$ animals.

\section{Experiment 3: Food Place Conditioning}

Figure 3 (upper panel) shows the results of the food place conditioning study. A two-way ANOVA disclosed a significant main effect for morphine treatment $(\mathrm{F}(1,27)=16.21, p<0.001)$, and further analysis revealed that $\mathrm{P}$ rats expressed a significantly greater preference for the food-paired chamber than $\mathrm{M}$ rats. Comparison analysis
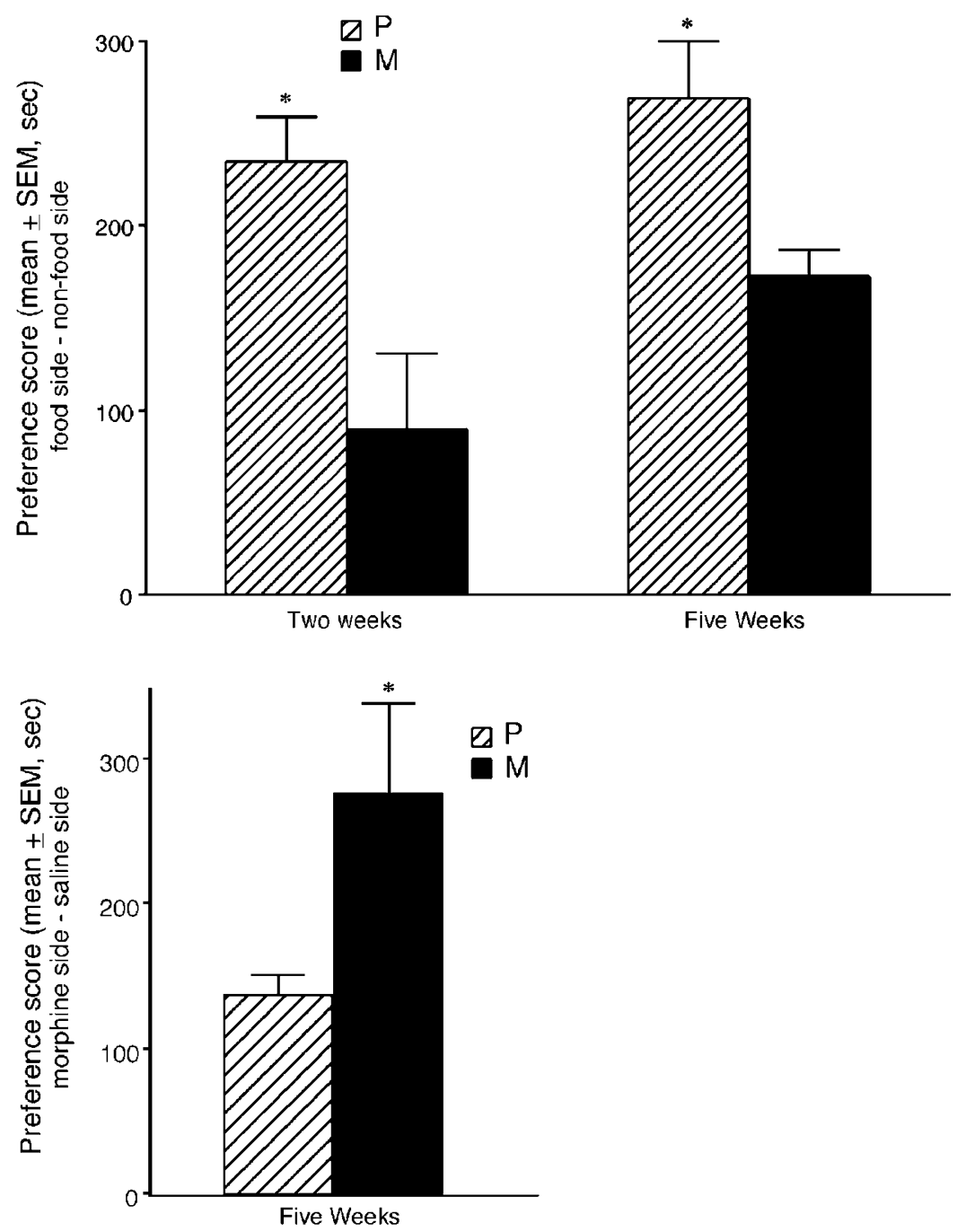

Figure 3 Upper panel: Preference scores for the food-paired environment expressed as the mean time in seconds spent in the food-paired side minus the mean time in seconds spent in the nonfood paired side on the test day. Weeks 2 and 5 refer to the number of weeks of morphine abstinence at the time of testing: *Significantly different $(p<0.0 \mathrm{I})$. Lower panel: Preference scores for the morphine-paired environment expressed as the mean time in seconds spent in the morphine-paired side minus the mean time in seconds spent in the saline-paired side on the test day: *Significantly different $(p<0.0 \mathrm{I})$. 
of each group pre- and postconditioning indicated that all groups except the 2-week $M$ group showed a significant place preference $(p<0.001)$. A comparison of body weights in the 2-week groups revealed no significant difference between $M$ and $P$ animals, indicating that $M$ rats were physically recovered from withdrawal and eating normally. In a previous study (Harris and Aston-Jones, 2002), we found that nonconditioned $\mathrm{P}$ and $\mathrm{M}$ groups given saline injections for three days paired with both compartments did not show a significant place preference $(p=0.12$; $\mathrm{F}(1,24)=2.4$, indicating that the place preference obtained in this study was not because of a side bias.

Figure 3 (lower panel) shows the results of morphine place conditioning in rats withdrawn at 5 weeks. Both $\mathrm{M}$ and $\mathrm{P}$ groups showed significant preferences for the morphine-paired environment $(\mathrm{F}(1,10)=47.17$ for $\mathrm{M}$ and 52.81 for $\mathrm{P}, p<0.001)$, but $\mathrm{M}$ animals showed significantly greater preference than $\mathrm{P}$ animals $(\mathrm{F}(1,10)=14.65$, $p<0.01)$. An analysis of locomotor activity on the test day revealed no significant differences in activity in either the morphine paired side $(p=0.61)$ or during the entire session $(p=0.09)$.

\section{DISCUSSION}

These experiments indicate that during protracted abstinence withdrawal from morphine animals are slower to learn about environmental cues that predict food reinforcement, while at the same time they readily learn about cues predictive of morphine reward. The $\mathrm{M}$ animals were significantly slower to learn the operant response for food reward, and were significantly slower to increase their responding when the response requirements for food were elevated. When tested at 2 weeks postwithdrawal, $M$ rats failed to show a place preference for a food reward, and at 5 weeks postwithdrawal $M$ animals developed a food place preference that was significantly smaller than the preference expressed by $\mathrm{P}$ animals. In contrast, when tested at 5 weeks postwithdrawal $M$ animals showed significantly greater morphine place preference than $\mathrm{P}$ animals, and also showed significantly greater retention of learning in the conditioned suppression paradigm.

The fact that $M$ animals were slower to learn an operant food reward task and failed to prefer an environment associated with food reward probably does not reflect a lack of desire for food. All rats in the morphine groups consumed all of the food they were given in both experiments. In addition, once the morphine-abstinent $M$ subjects learned an FR schedule in the instrumentalconditioning task, they obtained as many reinforcers in a session as the nonmorphine-exposed $\mathrm{P}$ rats. Furthermore, the body weights of the $M$ rats were not significantly different from those of $\mathrm{P}$ animals, and $\mathrm{M}$ rats appeared to be completely recovered from the experience of opiate withdrawal by the time of testing.

$M$ rats were slower to learn the association between bar pressing and receiving food. They were also slower to adapt when the response requirements for food were increased. It seems unlikely that their sluggishness in learning the elevated FR schedules were because of motor impairments because when they eventually learned the more demanding task requirements, they responded at rates similar to those seen in $\mathrm{P}$ animals. In addition, $\mathrm{M}$ rats did not have any apparent physical or behavioral abnormalities nor were they less active or less exploratory than $\mathrm{P}$ animals.

In the food place-conditioning experiment, 2-week-withdrawn $M$ rats showed no preference for the food-paired environment. The 5-week-withdrawan $M$ group developed a preference for the food-associated chamber that was much smaller than that found in $\mathrm{P}$ animals. All rats (including the morphine-abstinent rats) eagerly consumed all of the food reward immediately upon being placed in the food chamber, indicating that they found the food palatable. One possible explanation for the difference between $M$ and $P$ rats in food preference conditioning is that prior morphine exposure may alter the valence of natural reinforcers. It has been postulated that drugs of abuse alter the brain mechanisms involved in reward regulation and that these powerful neuropharmacological effects could 'short circuit' the neurochemistry of reward (Koob and Le Moal, 2001). Previous studies have also reported a decrease in the motivation for natural reinforcers, following amphetamine withdrawal (Barr and Phillips, 1999; Barr et al, 1999). In the opponent-process view (Solomon and Corbit, 1974), these reward systems are thought to become downregulated with chronic drug exposure to compensate for continuous stimulation of reward-related receptors during drug exposure. When the drug is removed during abstinence, this 'antireward' response is uncovered and leads to symptoms of anhedonia, depression, and anxiety. Continued drug use is thought to be rewarding not only because it stimulates the natural reward circuitry, but also because of the reinforcement associated with offsetting the antireward response (including stress hyper-responsivity and anxiety, as discussed above).

In support of this hypothesis, we found that $M$ animals withdrawn at 5 weeks showed much larger preferences for the morphine-associated environment than $\mathrm{P}$ animals. This replicates our previous reports of increased morphineassociated preference at 2 or 5 weeks postwithdrawal (Harris and Aston-Jones, 2001, 2002). In the nondependent $P$ subjects, the preference for the food-associated chamber was twice as high as that found for the morphine-associated chamber. The exact opposite was true for the $M$ animals, in which the preference for morphine-associated cues was twice as that found for food-associated cues. At 5 weeks postwithdrawal, the lack of motivation for food-reinforced responding seems to be diminishing, possibly indicating recovery of normal reward function. However, the motivation to seek out morphine-associated environmental cues has not decreased. This indicates that the changes that occur in the reward mechanisms following opiate exposure may involve multiple systems that recover at different rates.

In a theory outlined by Di Chiara (1998, 1999), natural reinforcers release DA in the accumbens shell and this response is thought to rapidly habituate. It is further proposed that the release of DA produced by drugs such as morphine does not habituate, thereby leading to a relative strengthening of the connections between environmental stimuli and drug reward. Such a mechanism may be involved in the altered responsiveness to morphine- $v s$ food-paired stimuli that we observed. For example, morphine pretreatment may have sensitized animals to 
the reinforcing effects of morphine, thereby leading to increased preference for the morphine-paired environment. Although we did not see evidence of locomotor sensitization in the $\mathrm{M}$ group on the morphine place-conditioning test day, others have shown that morphine pretreatment can lead to a sensitization to the reinforcing effects of morphine using a place-conditioning paradigm (Shippenberg et al, 1996; Shippenberg and Elmer, 1998).

Unlike the impaired performance in the instrumental tasks, $\mathrm{M}$ animals showed better performance than $\mathrm{P}$ rats in the conditioned suppression experiments. Thus, $\mathrm{M}$ rats displayed heightened retention of the tone-shock pairings, and a majority of $\mathrm{M}$ animals did not even resume responding during the entire $30 \mathrm{~min}$ session whereas this did not occur for any $\mathrm{P}$ rat. These findings indicate that $\mathrm{M}$ animals are not impaired in learning in general. We speculate that the enhanced learning shown in this paradigm by $M$ animals may be because of heightened stress reactivity and anxiety. Previously, we found that morphine-abstinent animals show prolonged augmented anxiety-like behaviors and enhanced retention of this behavior using a conditioned burying paradigm (Harris and Aston-Jones, 1993, 2001).

Furthermore, it has been well documented in humans that former opiate addicts show prolonged hyper-responsiveness to stress and that the hypothalamic-pituitary-adrenal axis is altered causing augmented release of corticotropinreleasing factor (CRF) and adrenocorticotropin hormone (Kreek, 1987; Kreek and Koob, 1998). This altered responsiveness to stress has been hypothesized to facilitate relapse in human addicts (Kreek, 1973, 1987; Childress et al, 1987; Kreek and Koob, 1998). In animals, exposure to stress has been shown to reinstate extinguished opiate self-administration (Shaham et al, 2000; Shalev et al, 2002), further establishing the link between stress and relapse to drugseeking behaviors. Increases in CRF transmission in both the amygdala and bed nucleus of the stria terminalis (BNST) have also been reported following opiate withdrawal (Kreek and Koob, 1998; Sarnyai et al, 2001). Increases in both norepinephrine and CRF in the BNST, and CRF in the amygdala, have been associated with the anxiety-like behaviors (Lee and Davis, 1997; Cecchi et al, 2002) and specifically with opiate withdrawal-induced aversions (Heinrichs et al, 1995; Delfs et al, 2000). These findings may explain the increased anxiety and greater retention of fear-associated contextual memories that accompany opiate abstinence.

Withdrawal from excessive opiate exposure appears to alter the motivational properties of natural reinforcers. It can lead to decrements in the motivation to acquire other appetitive reinforcers and increase anxiety levels. These results indicate some of the severe problems that addicts face during attempts to maintain drug abstinence. A better understanding of the brain mechanisms that underlie these changes in affective processing could lead to the development of more successful treatments for opiate addiction.

\section{ACKNOWLEDGMENTS}

This work was supported by PHS Grant DA-06214.

\section{REFERENCES}

Barr AM, Fiorino DF, Phillips AG (1999). Effects of withdrawal from an escalating dose schedule of $D$-amphetamine on sexual behavior in the male rat. Pharmacol Biochem Behav 64: 597-604.

Barr AM, Phillips AG (1999). Withdrawal following repeated exposure to $\mathrm{D}$-amphetamine decreases responding for a sucrose solution as measured by a progressive ratio schedule of reinforcement. Psychopharmacology 141: 99-106.

Cecchi M, Khoshbouei H, Javors M, Morilak DA (2002). Modulatory effects of norepinephrine in the lateral bed nucleus of the stria terminalis on behavioral and neuroendocrine responses to acute stress. Neuroscience 112: 13-21.

Childress AR, McLellan AT, Natale M, O’Brien CP (1987). Mood states can elicit conditioned withdrawal and craving in opiate abuse patients. NIDA Res Monogr 76: 137-144.

Delfs JM, Zhu Y, Druhan JP, Aston-Jones G (2000). Noradrenaline in the ventral forebrain is critical for opiate withdrawal-induced aversion. Nature 403: 430-434.

Di Chiara G (1998). A motivational learning hypothesis of the role of mesolimbic dopamine in compulsive drug use. J Psychopharmacol 12: 54-67.

Di Chiara G (1999). Drug addiction as dopamine-dependent associative learning disorder. Eur J Pharmacol 375: 13-30.

Dole VP, Nyswander ME, Kreek MJ (1966). Narcotic blockade. Arch Intern Med 118: 304-309.

Gerra G, Calbiani B, Zaimovic A, Sartori R, Ugolotti G, Ippolito L et al (1998). Regional cerebral blood flow and comorbid diagnosis in abstinent opioid addicts. Psychiatry Res 83: 117126.

Gold LH, Stinus L, Inturrisi CE, Koob GF (1994). Prolonged tolerance, dependence and abstinence following subcutaneous morphine pellet implantation in the rat. Eur J Pharmacol 253: $45-51$.

Harris GC, Aston-Jones G (1993). $\beta$-adrenergic antagonists attenuate withdrawal anxiety in cocaine- and morphine-dependent rats. Psychopharmacology 113: 131-136.

Harris GC, Aston-Jones G (2001). Augmented accumbal serotonin levels decrease the preference for a morphine associated environment during withdrawal. Neuropsychopharmacology 24: 75-85.

Harris GC, Aston-Jones G (2003). Enhanced morphine preference following prolonged abstinence: association with increased Fos expression in the extended amygdala. Neuropsychopharmacology 28: 292-299. http://www.acnp.org/citations/Npp071102346.

Heinrichs SC, Menzaghi F, Schulteis G, Koob GF, Stinus L (1995). Suppression of corticotropin-releasing factor in the amygdala attenuates aversive consequences of morphine withdrawal. Behav Pharmacol 6: 74-80.

Jaffe JH (1990). Drug addiction and abuse. In: Gilman AG, Rall TW, Nies AS, Taylor P (eds). Goodman and Gilman's The Pharmacological Basis of Therapeutics. Pergamon: New York. pp 522-573.

Koob GF, Le Moal M (1997). Drug abuse: Hedonic homeostatic dysregulation. Science 278: 52-58.

Koob GF, Le Moal M (2001). Drug addiction, dysregulation of reward, and allostasis. Neuropsychopharmacology 24: 97-129.

Kreek MJ (1973). Medical safety and side effects of methadone in tolerant individuals. J Am Med Assoc 223: 665-668.

Kreek MJ (1987). Multiple drug abuse patterns and medical consequences. In: Meltzer HY (ed). Psychopharmacolgy: The Third Generation of Progress. Raven Press: New York. pp 15971604.

Kreek MJ, Koob GF (1998). Drug dependence: stress and dysregulation of brain reward pathways. Drug Alcohol Depend 51: 23-47.

Krystal JH, Woods SW, Kosten TR, Rosen MI, Seibyl JP, van Dyck CC et al (1995). Opiate dependence and withdrawal: preliminary 
assessment using single photon emission computerized tomography (SPECT). Am J Drug Alcohol Abuse 21: 47-63.

Lee Y, Davis M (1997). Role of the hippocampus, the bed nucleus of the stria terminalis, and the amygdala in the excitatory effect of corticotropin-releasing hormone on the acoustic startle reflex. J Neurosci 17: 6433-6446.

Martin WR, Jasinski DR (1969). Physiological parameters of morphine dependence in man-tolerance, early abstinence, protracted abstinence. J Psychiatry Res 7: 9-17.

Ornstein TJ, Iddon JL, Baldacchino AM, Sahakian BJ, London M, Everitt BJ et al (2000). Profiles of cognitive dysfunction in chronic amphetamine and heroin abusers. Neuropsychopharmacology 23: 113-126.

Sarnyai Z, Shaham Y, Heinrichs SC (2001). The role of corticotropin-releasing factor in drug addiction. Pharmacol Rev 53: 209-243.

Shaham Y, Erb S, Stewart J (2000). Stress-induced relapse to heroin and cocaine seeking in rats: a review. Brain Res Rev 33: 13-33.

Shalev U, Grimm JW, Shaham Y (2002). Neurobiology of relapse to heroin and cocaine seeking: a review. Pharmacol Rev 54: 1-42.

Shippenberg TS, Elmer GI (1998). The neurobiology of opiate reinforcement. Crit Rev Neurobiol 12: 267-303.

Shippenberg TS, Heidbreder C, LeFevour A (1996). Sensitization to the conditioned rewarding effects of morphine: pharmacology and temporal characteristics. Eur J Pharmacol. 299: 33-39.

Solomon RL (1977). The opponent-process theory of acquired motivation: the affective dynamics of addiction. In: Maser JD, Seligman MEP (eds). Psychopathology: Experimental Models. WH Freeman and Co.: San Francisco. pp 124-145.

Solomon RL, Corbit JD (1974). An opponent-process theory of motivation: I. Temporal dynamics of affect. Psychol Rev 81: 119145.

Yoburn BC, Chen J, Huang T, Inturrisi CE (1985). Pharmacokinetics and pharmacodynamics of subcutaneous morphine pellets in the rat. J Pharmacol Exp Ther 235: 282-286. 